\title{
LOW RATES OF VITAMIN D SCREENING: CONCERN FOR AGING WITH POOR SKELETAL HEALTH IN THE OLDER MINORITIES
}

\author{
N.J. Rianon ${ }^{1}$, K.P. Murphy', C.B. Dyer ${ }^{1}$, B.J. Selwyn ${ }^{2}$
}

\begin{abstract}
Background: Regardless of discussion about vitamin D deficiency in minority population, there is a scarcity of information on vitamin D screening practice in older minority patients by their PCPs. Screening for risk factors does improve diagnosis and treatment of compromised skeletal health. Objective: To compare vitamin D screening rates between older patients from Caucasian and other non-Caucasian backgrounds including Blacks and Hispanics who were being treated by their PCPs for osteopenia, osteoporosis or related fractures. Design: Retrospective cross-sectional analysis. Setting: Electronic medical chart review from two urban primary care clinics (family medicine and geriatrics, Houston, TX) between January 2010 and December 2011. Participants: 133 patients 50 years or older who visited primary care clinics for osteopenia, osteoporosis or related fractures. Measurements: an order for 25-hydroxy vitamin D a year before or after an osteoporosis related visit. Results: Regardless of the clinic type, higher percentages of minority patients were not screened for vitamin D. While patients with older age from both groups were more likely to be screened, no single patient characteristic remained significant after adding clinic type to the logistic models. Conclusions: Lower rates of vitamin D screening put older minority patients at higher risk of aging with worsening skeletal health. Perspective and knowledge about vitamin D screening by PCP is recommended for future research to improve vitamin D screening and treatment in minority elderly.
\end{abstract}

Key words: Vitamin D screening, Racial/ethnic minority, primary care clinics, osteoporosis.

\section{Introduction}

In the era of controversy and misconception about serum vitamin D levels and screening, current peer reviewed scientific studies support a recommendation for determining and maintaining optimum vitamin $\mathrm{D}$ levels for appropriate management of osteoporosis and related fractures in at risk older patients (1-2). Minority groups including Blacks and Hispanics are at risk of bone loss and osteoporosis due to high prevalence of vitamin $\mathrm{D}$ deficiency among them (3-7). Yet, most discussions about risk factors of age-related bone loss and need for its appropriate prevention and treatment are focused on Caucasians. Thus the knowledge gap in providers for minority skeletal health risk factors remains large (7-10).

Vitamin D is an essential nutrient for skeletal health and plays an important role in prevention and

1. Division of Geriatric and Palliative Medicine, Department of Internal Medicine, University of Texas Medical School at Houston, TX, USA; 2. Division of Epidemiology, University of Texas Houston School of Public Health, TX, USA

Corresponding Author: Nahid Rianon, MD, DrPH, Assistant Professor of Geriatric and Palliative Medicine, Department of Internal Medicine, University of Texas Medical School at Houston, TX, 6431 Fannin St. \#MSB 5.120, Houston, Texas 77030, E-mail: Nahid.J.Rianon@uth.tmc.edu, Telephone: 713-500-6317, Fax: 713500-0706 treatment of age-related osteoporosis and associated fractures $(1,3-5,11-13)$. While inadequate treatment of osteoporosis in older patients by primary care providers $(\mathrm{PCP})$ is a known public health concern, low rates of screening by PCPs were also reported in older patients with compromised skeletal health (14). Non-Caucasian minorities with darker skin are at greater risk of vitamin $\mathrm{D}$ deficiency as they require more exposure to ultraviolet light to produce the same level of vitamin D as the Caucasians with lighter skin (5). The biological risk of vitamin D deficiency makes it more important to screen and monitor vitamin $\mathrm{D}$ in older minority patients with darker skin (e.g., Blacks and Hispanics) being treated for osteoporosis. Regardless of discussion about vitamin D deficiency in minority population, there is a scarcity of information on vitamin D screening practice in older minority patients by their PCPs. Screening for risk factors does improve diagnosis and treatment of compromised skeletal health (14). We compare vitamin D screening rates between older patients from Caucasian and other non-Caucasian backgrounds including Blacks and Hispanics who were being treated by their PCPs for osteopenia, osteoporosis or related fractures. 


\section{Methods}

Retrospective data were collected by electronic medical chart review for a cross-sectional analysis. Data consisted of 133 patients, 50 years or older who were seen in two urban primary care clinics (family medicine and geriatrics, Houston, TX) for osteopenia, osteoporosis and associated fractures by their PCPs between January 2010 and December 2011. Racial/ethnic background was recorded in the medical record during clinic visits. Caucasians, African Americans, Hispanics, and others (Asians and Native Indians) were the original racial/ ethnic backgrounds reported in the medical records. For the purposes of this study, based on greater risk of vitamin D deficiency in minorities (non-Caucasian/ non-light skin), we decided to report our results by two groups: Caucasian (White) and Non-Caucasian minority African American, Hispanics, Native Indians and Asians) for the current analysis. (As per medical chart, we used the term "African American" which is otherwise mentieond as "Black" when referring to previous studies.)

Descriptive patient characteristics included: age, body mass index (BMI), current smoking and/ or alcohol use, commonly reported chronic diseases, e.g., hypertension, type 2 diabetes, arthritis and depression. An order for 25 hydroxy vitamin $\mathrm{D}$ levels in the medical record one year before or after the osteoporosis related visit date was considered positive for vitamin D screening. Data analysis included descriptive statistics of patients in the Caucasian and minority groups. A bivariate analysis (done separately for Caucasian and non-Caucasian minority groups) described associations between
Vitamin D screening status (yes or no) and age, BMI, smoking, alcohol and reported chronic diseases. Chi square or logistic regression was used to determine if any association was statistically significant at $p$ value of $<0.05$. Separate bivariate analyses compared differences in vitamin D screening by clinic type (family medicine vs. geriatrics clinics) for patients from each Race/ ethnic background. Separate forward stepwise logistic regressions were conducted to determine indicators of vitamin D screening for Caucasian and non-Caucasian minority groups. Variables (age, hypertension and clinic type) significant at $p<0.10$ from the first bivariate analysis were included in the regression model. Age was kept as a continuous variable; having a diagnosis of hypertension and being seen in a family medicine clinic were considered high risk levels for the "hypertension" and "clinic type" variables in the logistic model. Results were reported in odds ratio with $95 \%$ confidence intervals.

\section{Results}

There were no statistically significant differences in patient characteristics including demographics, behavioral risk factors, co-morbidities, supplements and medication use for promoting bone health except for alcohol use between the Caucasian and non-Caucasian Minority groups (Table 1). The mean age for the entire sample $(\mathrm{N}=133)$ was $71 \pm 13$ years with a range of $51-97$ years. Most patients were Caucasians $(63 \%)$, women $(93 \%)$, and reported no current smoking $(85 \%)$ or alcohol use $(64 \%)$.

For Caucasian patients, significant association $(p<0.05)$

Table 1

Patient characteristics by Race/Ethnic backgrounds

\begin{tabular}{lccc}
\hline Patient characteristics & Caucasians [N=84 (63\%)] & Minorities [N=49, (37\%)] & p value \\
\hline Age (mean \pm SD) years & $70 \pm 13$ & $71 \pm 12$ & 0.53 \\
Gender (Female) & $77(92 \%)$ & $47(96 \%)$ & 0.48 \\
BMI (mean \pm SD) kg/m² & $25 \pm 6$ & $26 \pm 5$ & 0.66 \\
Vitamin D screening was done (Yes) & $32(38 \%)$ & $17(35 \%)$ & 0.69 \\
Clinic type (Family Medicine) & $38(45 \%)$ & $23(45 \%)$ & 0.85 \\
Not a current smoker & $46(81 \%)$ & $27(93 \%)$ & 0.20 \\
No current use of alcohol & $27(54 \%)$ & $22(81 \%)$ & 0.02 \\
Hypertension (Yes) & $37(44 \%)$ & $20(41 \%)$ & 0.72 \\
Type 2 Diabetes (Yes) & $7(8 \%)$ & $6(12 \%)$ & 0.46 \\
Depression (yes) & $18(21 \%)$ & $12(24 \%)$ & 0.68 \\
Arthritis (Yes) & $10(12 \%)$ & $8(16.33)$ & 0.47 \\
Use bone promoting medication (Yes) & $49(58 \%)$ & $28(57 \%)$ & 0.89 \\
Vitamin D deficiency (Yes) & $13(42 \%)$ & $8(47 \%)$ & 0.73 \\
\hline
\end{tabular}

Notes: $\mathrm{SD}=$ standard deviation, $\mathrm{BMI}=$ body mass index 
Table 2

Association between vitamin D screening status (yes and no) and other patient risk factors for Caucasian and Minority groups

\begin{tabular}{|c|c|c|c|c|c|c|c|}
\hline \multirow{3}{*}{\multicolumn{2}{|c|}{ Patient characteristics }} & \multicolumn{2}{|c|}{ Caucasian $($ Race $=0)(\mathrm{N}=84)$} & \multirow{3}{*}{$\begin{array}{l}\text { P value } \\
\mathbf{N}(\%)\end{array}$} & \multicolumn{2}{|c|}{ Minority $($ Race $=1)(\mathrm{N}=49)$} & \multirow[t]{3}{*}{ P value } \\
\hline & & \multirow{2}{*}{$\begin{array}{c}\text { Vitamin } D \\
\text { screening was } \\
\text { done }(\mathrm{N}=32) \\
\mathrm{N}(\%)\end{array}$} & \multirow{2}{*}{$\begin{array}{c}\text { Vitamin D } \\
\text { screening } \\
\text { was not done } \\
(\mathrm{N}=52) \\
\mathrm{N}(\%)\end{array}$} & & \multirow{2}{*}{$\begin{array}{c}\text { Vitamin D } \\
\text { screening was } \\
\text { done }(\mathrm{N}=17) \\
\mathrm{N}(\%)\end{array}$} & \multirow[t]{2}{*}{$\begin{array}{c}\text { Vitamin D } \\
\text { screening } \\
\text { was not done } \\
(\mathrm{N}=32)\end{array}$} & \\
\hline & & & & & & & \\
\hline Age (yea & & $75.84 \pm 12.16$ & $66.44 \pm 12.36$ & 0.002 & $76.82 \pm 9.99$ & $68.56 \pm 11.92$ & 0.025 \\
\hline Gender & $M=1$ & $4(12.50)$ & $3(5.77)$ & 0.419 & $1(5.88)$ & $1(3.13)$ & 1.00 \\
\hline & $\mathrm{F}=2$ & $28(87.50)$ & $49(94.23)$ & & $16(94.12)$ & $31(96.88)$ & \\
\hline \multirow[t]{2}{*}{ Clinics } & FM & $7(21.88)$ & $31(59.62)$ & 0.001 & $5(29.41)$ & $18(56.25)$ & 0.073 \\
\hline & GM & $25(78.13)$ & $21(40.38)$ & & $12(70.59)$ & $14(43.75)$ & \\
\hline \multicolumn{2}{|c|}{ Had a diagnosis of hypertension } & $22(68.75)$ & $25(48.08)$ & 0.064 & $9(52.94)$ & $20(62.50)$ & 0.517 \\
\hline \multicolumn{2}{|c|}{ Had a diagnosis of Type 2 diabetes } & $3(9.38)$ & $4(7.69)$ & 0.786 & $2(11.76)$ & $4(12.50)$ & 0.940 \\
\hline \multicolumn{2}{|c|}{ Had a diagnosis of depression } & $22(68.75)$ & $44(84.62)$ & 0.105 & $13(76.47)$ & $24(75.00)$ & 1.000 \\
\hline \multicolumn{2}{|c|}{ Had a diagnosis of arthritis } & $4(12.50)$ & $6(11.54)$ & 1.000 & $3(17.65)$ & $5(15.63)$ & 1.000 \\
\hline \multicolumn{2}{|c|}{ Taking Calcium and vitamin D supplementation } & $2(6 \%)$ & $3(6 \%)$ & 0.928 & $1(6 \%)$ & $4(12.5 \%)$ & 0.466 \\
\hline
\end{tabular}

Table 3

Vitamin D screening (yes and no) by clinic types (family medicine vs. geriatrics) in Caucasians and minority groups

\begin{tabular}{lccccc}
\hline & \multicolumn{2}{c}{ Caucasian $(\mathbf{N}=84)$} & p value & \multicolumn{2}{c}{ Minority (N=49) } \\
Vitamin D & Family Medicine & Geriatrics & & Family Medicine & Geriatrics \\
\hline Screening was done (yes) & $7(18.42)$ & $25(54.35)$ & 0.001 & $5(21.74)$ & $12(46.15)$ \\
Screening not done (no) & $31(81.58)$ & $21(45.65)$ & & $18(78.26)$ & $14(53.85)$ \\
\hline
\end{tabular}

Table 4

Indicators of not screening for vitamin D in Caucasians and minority groups: results from two logistic regression models

\begin{tabular}{|c|c|c|c|c|}
\hline Indicators & \multicolumn{2}{|c|}{ Caucasians } & \multicolumn{2}{|c|}{ Minority groups } \\
\hline \multirow[t]{2}{*}{ Age } & 0.95 & 0.98 & 0.93 & 0.93 \\
\hline & $(0.91 \text { to } 0.99)^{*}$ & (0.93 to 1.04$)$ & $(0.87 \text { to } 0.99)^{*}$ & (0.86 to 1.02$)$ \\
\hline Hypertension (Yes/No) & (0.25 to 1.89$)$ & (0.21 to 1.73$)$ & (0.58 to 8.46$)$ & (0.58 to 8.67$)$ \\
\hline \multirow[t]{2}{*}{ Clinic type (FM vs. GM) } & ----------- & 3.30 & ------------- & 1.13 \\
\hline & & (0.79 to 13.84 & & (0.16 to 7.83$)$ \\
\hline
\end{tabular}

${ }^{*} \mathrm{P}<0.05 . \mathrm{FM}=$ family medicine clinic; $\mathrm{GM}=$ geriatrics clinic. Each determinant variable is adjusted for all others in the model. 
was noted between vitamin D screening status and clinic type, and age (Table 2). Among the Caucasian patients, more patients not screened for Vitamin D were seen in family medicine clinic $(60 \%)$ than in geriatric clinic $(40 \%)$. (Table 2). For minority population, age was the only variable showing a significant positive association with getting vitamin D screening (Table2).

When checked by type of clinic (Family Medicine vs. Geriatrics), screening was significantly lower for patients seen in family medicine clinic regardless of Ethnic/Racial background, while a higher percentage of Caucasian patients seen in geriatrics clinic were screened for vitamin $\mathrm{D}$ than those not screened. For minority groups, more patients were not screened for vitamin D compared to those screened regardless of where they were seen (Family Medicine vs. Geriatrics) (Table 3).

Older age was significantly associated with positive screening status for vitamin $\mathrm{D}$ in the logistic regression model for both Caucasian and minority patients (Table 4). While older patients from both groups were more likely to be screened for vitamin $\mathrm{D}$, no single patient characteristic remained significant after adding clinic type to the logistic models (Table 4).

\section{Discussions}

Older patients from minority racial/ethnic groups have higher odds of not getting vitamin D screening when seen in primary care clinics for compromised bone health, e.g., osteopenia, osteoporosis and related fractures. While significantly greater percentage $(54 \%)$ of Caucasian patients were being screened for vitamin $\mathrm{D}$ in the geriatric clinics, non-Caucasian minority patients had a lower rate of vitamin D screening regardless of their clinic type (geriatrics vs. family medicine) (Table 3).

Despite having multiple risk factors for bone loss including age, the diagnosis of having either osteopenia or osteoporosis or related fractures, and having darker skin, minority patients in our study remain at great danger for not being screened for vitamin $\mathrm{D}(5,15)$. Management of osteoporosis remains incomplete without required supplementation of vitamin $\mathrm{D}$ which may not be possible without knowing the patients' vitamin D levels (16). Regardless of their race/ethnic background, very few patients were noted to take calcium and vitamin D supplementation in our study. Due to the nonprescription nature of these supplements, it is difficult to accurately confirm this information. Nonetheless, based on the electronic chart review, vitamin D screening is low in the at risk patient population in our study.

Improved cardiac ejection fraction after optimizing vitamin $\mathrm{D}$ level in minority (African American) men with vitamin D deficiency and increased c-reactive protein in patients with low vitamin $\mathrm{D}$ are reasons to maintain good vitamin D levels in patients with chronic disease such as hypertension and diabetes (1213). Presence of the two common co-morbidities of hypertension and diabetes in our study patients who also suffer from compromised skeletal health indicates need for vitamin D screening to promote healthy aging in this patient population. Although not statistically significant, a higher percentage of minority patients $(47 \%)$ had vitamin D deficiency (a level $<30 \mathrm{ng} / \mathrm{ml}$ ) (16-17) compared to their Caucasian counterpart (42\%) (Table 1). Case identification, screening and diagnosis can improve skeletal health outcomes in clinics which strategically designed and emphasized specific guidelines for improving osteoporosis treatment (14, 18-19). These clinics identified at-risk patients, screened and treated them for osteoporosis as appropriate (18-19). Evidence suggest that screening for vitamin $\mathrm{D}$ levels and treating any deficiency improves skeletal health in order to promote healthy aging in the minority older patients with multiple chronic disease (12-13).

Regardless of their race/ethnic backgrounds, more patients seen in family medicine clinic were not screened for vitamin D levels. Competing priorities of other chronic diseases managed by PCPs and time constraints for each visit often make it difficult for the PCP to address silent diseases like osteoporosis and skeletal health (20-23); and yet these are the older patients who are most in need of optimum vitamin D levels (12-13). While it may be that the PCPs in family medicine may consider vitamin D tests a specialty test, other issues may contribute to less testing by PCPs, such as, problem with insurance reimbursement, misconception about and lack of appropriate guidelines for screening, and lack of knowledge about current updates on vitamin D screening to maintain appropriate levels in patients with compromised skeletal health $(1,20-24)$.

Percentages of Caucasian and minority (Table 1) patients seen in family medicine and geriatric clinics were similar. Yet, more minorities were not screened in both clinics with significant differences in screening between Caucasians and minorities in the geriatric clinic (Table 3). Our results show lower prevalence of screening regardless of the clinic type where they are seen. While patient with older age (from both racial/ ethnic backgrounds) were more likely to be screened, the significant association between old age and vitamin D screening became non-significant after adding clinic type (family medicine vs. geriatrics clinic) to the regression model (table 4). Overall low rates of screening by family medicine had most likely influenced the outcome in the regression model. Despite having multiple risk factors, e.g., older age, concurrent diagnosis of cardio-metabolic co-morbidities in the presence of compromised skeletal health and having darker (non-light) skin, minority patients are not being screened for the essential vitamin D which can improve skeletal health outcomes (1617). Recent USPSTF recommendation against routine screening for vitamin $\mathrm{D}$ in general population may contribute to a low screening rate in general (2). Our results have led to educational interventions with a 
conscious effort to increase awareness of minority skeletal health in both family medicine and geriatric clinics.

In agreement with current scientific literature, our data indicate lower rates of vitamin D screening in minority older patients who suffer from multiple known risk factors that can be improved with optimum vitamin $D$ levels. Among those who were screened for vitamin D levels in both primary care clinics (family medicine and geriatrics clinics), higher percentages of minority patients had vitamin $\mathrm{D}$ deficiency compared to the Caucasian patients of similar age and co-morbidities. We recommend future studies aimed at determining and improving physician factors, e.g., knowledge and appropriate education, to improve vitamin D screening and supplementation in at-risk older minority patients with darker skin shades.

Acknowledgement: This work was supported by a grant from the Texas Academy of Family Physicians (TAFP) and Herzstein Foundation.

Conflict of interest: None.

Ethics standards: The study was approved by IRB of the University of Texas Houston Health Science Center.

\section{References}

1. Rosen C and Taylor C. Common misconceptions about vitamin Dimplications for clinicians. Nat Rev Endocrinol 2013; 9:434-438.

2. USPSTF (U.S. Preventative Services Task Force) report. http://www. uspreventiveservicestaskforce.org/uspstf/uspsvitd.htm. Accessed August 16, 2015.

3. Ginde A, Liu M, and Camargo Jr C. Demographic differences and trends of vitamin D insufficiency in the US population, 1988-2004. Arch Intern Med 2009; 169(6):626-632.

4. Powe C, Evans M, Wenger J, et al. Vitamin D-binding protein and vitamin D status of Black Americans and White Americans. N Engl J Med, 2013; 369(21):1991- 2000.

5. Weishaar T and Vergili JM. Vitamin D status is a biological determinant of health disparities. J Acad Nutr Diet 2013; 113(5):643-651.

6. Peiris AN, Bailey BA, Peiris P, Copeland R, Manning T. Race and Vitamin D Status and Monitoring in Male Veterans. J Natl Med Assoc 2011; 103,492-497.

7. Grant W and Peiris A. Possible Role of Serum 25-Hydroxyvitamin D in Black-White Health Disparities in the United States. J Am Med Dir Assoc 2010; 11:617-628
8. Benson J and Skull S. Hiding from the sun- vitamin D deficiency in refugees. Aust Fam Physician 2009; 36:355-357.

9. Brock K, Wilkinson M, Cook R, Lee S, Bermingham M. Associations with vitamin D deficiency in 'at risk" Australians. Journal of Steroid Biochemistry and Molecular Biology 2004; 89-90:581-588.

10. Cauley JA. Public Health Impact of Osteoporosis. J Gerontol A Biol Sci Med Sci 2013; 68(10):1243-1251.

11. Queally J, Kiernan C, Shaikh M, Rowan F, Shaikh M and Bennett D. Initiation of osteoporosis assessment in the fracture clinic results in improved osteoporosis management: a randomised controlled trial. Osteoporos Int 2013; 24:1089-1094.

12. Youssef D, El Abbassi AM, and Cutchins D. Vitamin D deficiency: Implications for acute care in the elderly and in patients with chronic illness. Geriatr Gerontol Int 2011; 11:395-407.

13. Zia A, Komolafe B, Moten M, Ahokas R, McGee J, Rosenberg W, Bhattacharya S and Weber K. Supplemental vitamin D and calcium in the management of African Americans with heart failure having hypovitaminosis D. Am J Med Sci 2011;341:113-118.i

14. Rianon N, Anand D and Rasu R. Changing trends in osteoporosis care from specialty to primary care physicians. Curr Med Res Opin 2013; 29(8):881-888.

15. National Osteoporosis Foundation (NOF). www.nof.org. Accessed August 16, 2015.

16. Rosen C, Abrams S, Aloia J, et al. IOM committee members respond to Endocrine Society vitamin D guideline. J Clin Endocrinol Metab 2012; 97:1146-1152.

17. Holick M, Binkley N, Bischoff-Ferrari H, et al. Guidelines for preventing and treating vitamin D deficiency and insufficiency revisited. J Clin Endocrinol Metab 2012; 97(4):1153-1158.

18. van Helden S, Cauberg E, Geusens P, Winkes B, van der Weijden T, and Brink P. The fracture and osteoporosis outpatient clinic: an effective strategy for improving implementation of an osteoporosis guideline. J Eval Clin Pract 2007; 13:801-805.

19. Dell R. Fracture Prevention in Kaiser Permanente in Southern California. Osteoporos Int 2011; 22(3):457-460.

20. Lewiecki EM. Managing osteoporosis: challenges and strategies. Cleve Clin J Med 2009; 76(8):457-466.

21. McNearney TA, Shepherd AJ, Chhabra A and Goel N. Primary care house staff attitudes toward osteoporosis management. South Med 2006; 99 (5):461466.

22. Simonelli C, Killeen K, Mehle S and Swanson L. Barriers to osteoporosis identification and treatment among primary care physicians and orthopedic surgeons. Mayo Clin Proc 2002; 77(4):334-338.

23. Solomon D, Connelly M, Rosen C, et al. Factors related to the use of bone densitometry: survey responses of 494 primary care physicians in New England. Osteoporos Int 2003; 14:123-129.

24. Rianon N, Murphy K, Guanlao R, Hnatow M, De Leon E and Selwyn B. Suboptimal vitamin D screening in older patients with compromised skeletal health. J Eval Clin Pract 2014; 20(2):144-148. 